\title{
Fermi surface studies of low-dimensional organic conductors based on BEDT-TTF
}

\author{
John Singleton ${ }^{\mathrm{a}, *}$, Jason Caulfield ${ }^{\mathrm{a}}$, Stephen Hill $^{\mathrm{a}}$, Stephen Blundell ${ }^{\mathrm{a}}$, \\ Wieslaw Lubczynski ${ }^{a}$, Andrew House ${ }^{a}$, William Hayes ${ }^{a}$, Jos Perenboom ${ }^{b}$, \\ Mohammedally Kurmoo ${ }^{\mathfrak{c}}$, Peter Day ${ }^{\mathfrak{c}}$ \\ a Department of Physics, University of Oxford, The Clarendon Laboratory, Parks Road, Oxford OXI 3PU, UK \\ ${ }^{b}$ High Field Magnet Laboratory and Research Institute for Materials, NL 6525 ED Nijmegen. The Netherlands \\ ${ }^{c}$ The Royal Institution, 21 Albemarle Street, London WIX 4BS, UK
}

\begin{abstract}
This paper provides an introduction to charge-transfer salts of the ion bis(ethylenedithio)tetrathiafulvalene (ET) and their band-structure, and reviews some recent experiments on the salts involving high magnetic fields carried out by the Oxford group and coworkers.
\end{abstract}

\section{Introduction}

The idea of making solids with band-structures tailored to particular applications has long fascinated physicists. Perhaps the best known example of this is the semiconductor superlattice; another approach is to use small organic molecules to build up solids with desirable metallic, semiconducting or even superconducting properties [1]. Much of the current work in this field centres on charge-transfer salts, systems in which a number of molecules jointly donate an electron to another ion (the anion), thereby producing a stable crystalline lattice $[2,3]$. Within this family of compounds charge-transfer salts of the ion bis(ethylenedithio)tetrathiafulvalene (BEDT-TTF or ET) [1-3] form a versatile system for the study of band formation; by varying the anion $X$ in the salt (ET) $X$, the stoichiometry (and hence the band shape) and band-filling may be adjusted. In this way metallic, semimetallic, and semiconducting ET salts have been made, some of which are also superconductors.

* Corresponding author.
Fig. 1 shows the crystal structure of $\mathrm{K}$-(ET) ${ }_{2} \mathrm{Cu}(\mathrm{NCS})_{2}$, and illustrates many of the typical structural properties of ET salts $[2,3]$. The ET molecules stack in two-dimensional (2D) planes, separated by layers of $\mathrm{Cu}(\mathrm{NCS})_{2}$ anions. Transfer of electrons between ET molecules occurs readily within the $2 \mathrm{D}$ planes, but much less so in the perpendicular direction; as a result, the band-structures and electrical properties of ET salts are rather 2D. Fig. 2 shows the Brillouin zone and Fermi surface (FS) [4] for $\kappa$-(ET $)_{2} \mathrm{Cu}(\mathrm{NCS})_{2}$ calculated with an extended Hückel (tight-binding) model. As in many ET salts, the FS consists of a quasi-1D open section (electrons) and a closed 2D hole pocket. The simplicity of the band-structures of ET salts, and the relative ease with which they may be calculated and measured makes the compounds attractive systems for studying many problems in Condensed Matter Physics [5].

In the following sections we shall briefly review some of the techniques involving high magnetic fields which are used to study the FS of ET salts, concentrating on recent experiments carried out by the Oxford group and coworkers. Further references giving details of the 


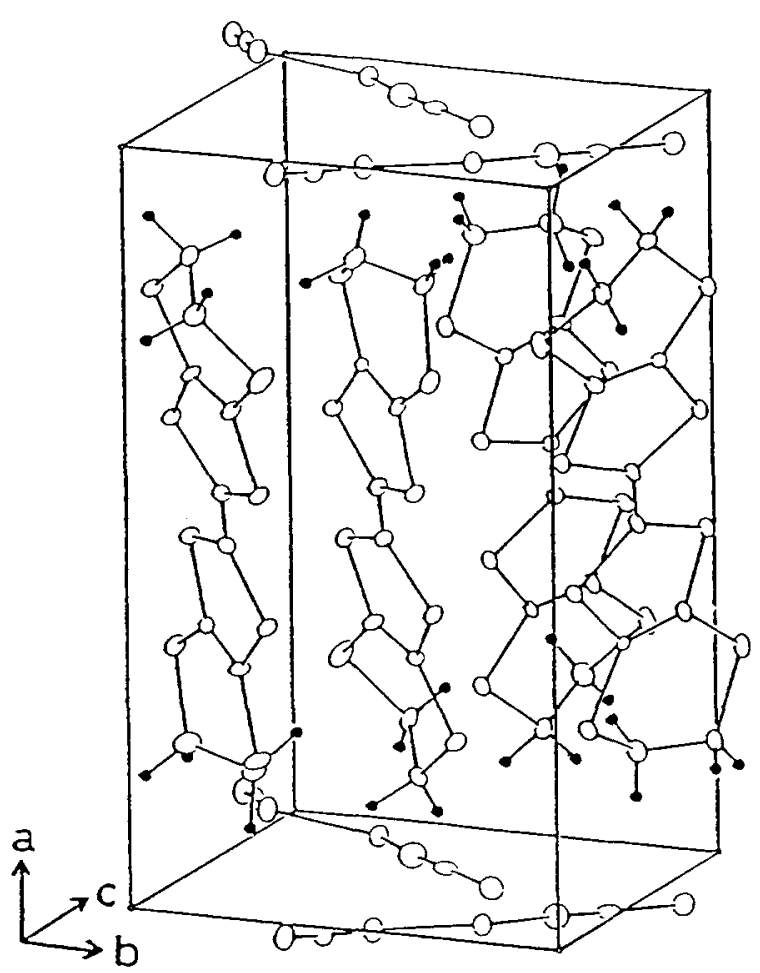

Fig. 1. Unit cell of $\kappa-(\mathrm{ET})_{2} \mathrm{Cu}(\mathrm{NCS})_{2}$, showing the stacking of the ET molecules between the anion planes [2].

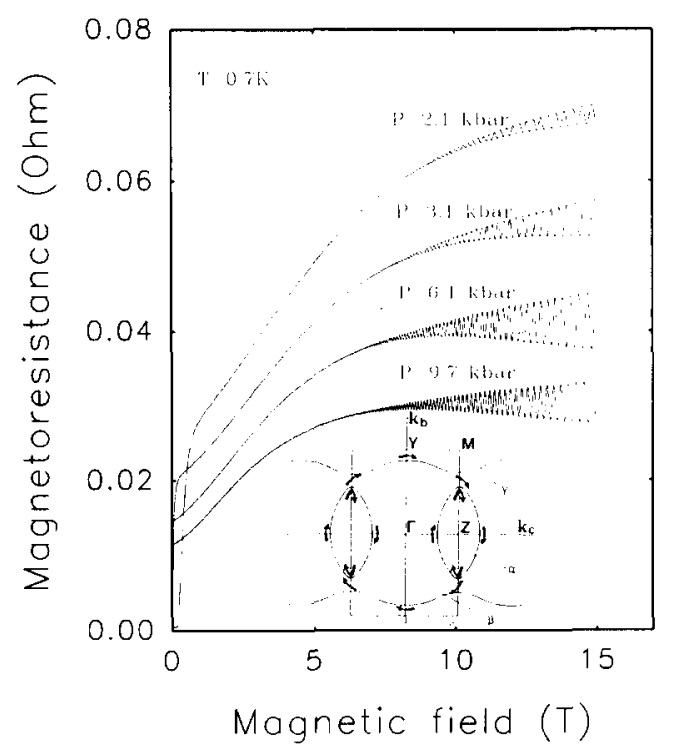

Fig. 2. Magnetoresistance of $\mathrm{K}-(\mathrm{ET})_{2} \mathrm{Cu}\left(\mathrm{NCS}_{2}\right.$ at $0.7 \mathrm{~K}$ (magnetic field applied perpendicular to the conducting $b c$ planes) for four different pressures [11]. The inset shows the Brillouin zone, Fermi surface and $\alpha$ - and $\beta$-orbits (after Ref. [7]). growth techniques used to prepare ET salts and other practical considerations are listed in Ref. [6].

\section{The Shubnikov-de Haas effect and magnetic break- down in $\kappa-(\mathrm{ET})_{2} \mathrm{Cu}(\mathrm{NCS})_{2}$}

Experimental tests of models for superconductivity require a knowledge of the density of states at the Fermi energy, and so a useful starting point is a material with a simple bandstructure. In this context ET salts are ideal; for example, $\kappa-(\mathrm{ET})_{2} \mathrm{Cu}(\mathrm{NCS})_{2}$ has the simple and readily calculated quasi-2D FS shown in Fig. 2, and for some time was the highest temperature organic superconductor $\left(T_{\mathrm{c}} \sim 10.4 \mathrm{~K}\right)[3-5]$.

Experimental studies of FS areas and associated effective masses in ET salts are often carried out using the Shubnikov-de Haas (SdH) effect [4-10]. Magnetoresistance (MR) data for $\kappa$-(ET) $)_{2} \mathrm{Cu}(\mathrm{NCS})_{2}$ are shown in Fig. 2 for a number of hydrostatic pressures [11]; one series of $\mathrm{SdH}$ oscillations, due to the $k$-space orbit around the 2D hole pocket (the $\alpha$-orbit; see inset) is plainly visible. The period of the oscillations gives the $k$-space area of the hole pocket, whilst the dependence of the oscillation amplitude on temperature and field may be fitted with the Lifshitz-Kosevich (LK) formula to give the effective mass and Dingle temperature [10]. Note also that the normal state conductivity of the sample increases with pressure and the upper critical field $B_{\mathrm{c} 2}$ decreases sharply.

As the pressure is raised further (Fig. 3) [11], a second series of oscillations with a higher frequency is observed in the MR, superimposed on the $\alpha$-orbit oscillations. Similar oscillations have been observed at $\sim 20 \mathrm{~T}$ at ambient pressure, and are attributed to magnetic breakdown [7-9]. Magnetic breakdown is due to electrons tunnelling between states of equal energy in adjacent sections of FS, thus describing a larger $k$-space orbit $[7-9,11]$. The inset in Fig. 2 indicates the primary breakdown orbit (the $\beta$-orbit) in $\kappa-\left(\mathrm{ET}_{2}\right) \mathrm{Cu}(\mathrm{NCS})_{2}$ around the outer edges of both the 2D and 1D FS sections.

The effective masses associated with the $\alpha$ and $\beta$ orbits were derived by fitting the $\mathrm{SdH}$ and magnetic breakdown oscillation amplitudes measured in the range $0.5-5 \mathrm{~K}$ at each pressure to the LK formula $[10,11]$ and are shown in Fig. 4; the behaviour of the two masses is almost identical in the regions where the breakdown effect can be resolved. Two distinct regions are observed: above a pressure $P_{\mathrm{c}} \sim 5 \mathrm{kbar}$ the rate of change in effective mass with pressure is some eight times smaller than below $P_{c}$. The variation of the superconducting $T_{\mathfrak{c}}$ with pressure (measured in the same experiments) and its disappearence at $P_{\mathrm{c}}$ (Fig. 4) appears to be connected with the rapid change in the effective mass in this region; it is thought 


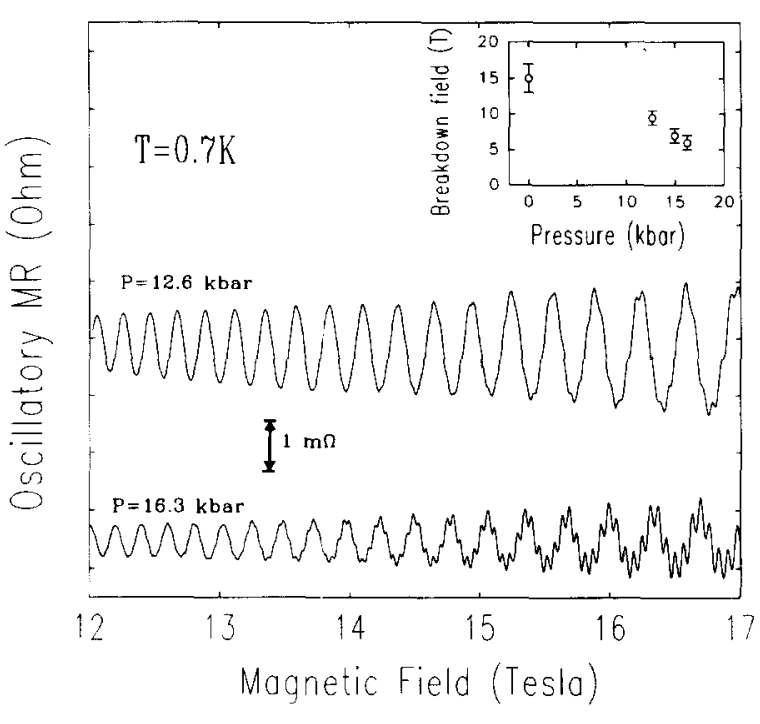

Fig. 3. The oscillatory part of the magnetoresistance of $\kappa$ $(\mathrm{ET})_{2} \mathrm{Cu}(\mathrm{NCS})_{2}$ above $12 \mathrm{~T}$ at $0.7 \mathrm{~K}$ (field applied perpendicular to the conducting planes) at two different pressures. The inset shows the characteristic breakdown field $B_{0}$ as a function of pressure [11].

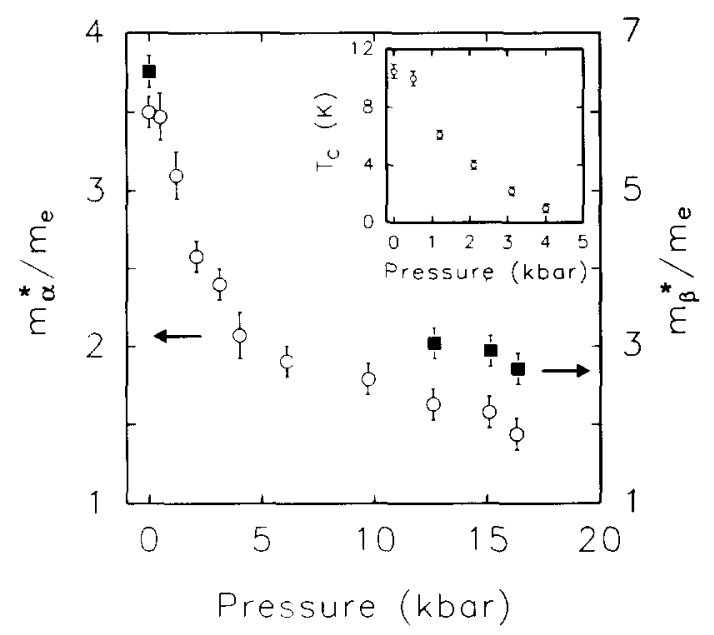

Fig. 4. The effective masses of the $\alpha$-orbit (hollow circles, left and vertical scale) and $\beta$-orbit (filled squares, right-hand vertical scale) of $\kappa$-(ET) $)_{2} \mathrm{Cu}(\mathrm{NCS})_{2}$ as a function of pressure. The inset shows the superconducting $T_{\mathrm{c}}$ versus pressure [11].

that the variation in $T_{\mathrm{c}}$ corresponds directly to changes in the electronic density of states at the FS associated with the changes in effective mass. Such measurements of the direct correspondence between superconducting properties and band-structure are now being used to test different possible models for superconductivity in ET salts $[11,12]$.

\section{The de Haas-van Alphen effect in the vortex state}

The de Haas-van Alphen (dHvA) effect in an oscillatory contribution to the magnetisation analogous to the $\mathrm{SdH}$ effect and provides a powerful tool for studying the FS of ET salts $[13,14]$. Interestingly, there have been clear demonstrations of dHvA oscillations in the vortex state of type II superconductors such as $\mathrm{NbSe}_{2}[15,16]$, $\mathrm{V}_{3} \mathrm{Si}[16]$ and $\mathrm{Nb}_{3} \mathrm{Sn}$ [17]. Recently such studies have been extended to $\kappa$-(ET) ${ }_{2} \mathrm{Cu}(\mathrm{NCS})_{2}$ [18]. The experiments were performed in a dilution refrigerator, using the field modulation method at temperatures down to $20 \mathrm{mK}$ [18]. The magnetic field was applied in $a b$ plane; the angle between the applied field and the $a$-axis ( $a$ is perpendicular to the $2 \mathrm{D}$ planes) is $\theta$.

A typical dHvA signal is shown in Fig. $5\left(\theta=29^{\circ}\right)$ [18]. Data in the normal state are in accord with previous studies [13]. Fig. 5 also shows a fit of the LK formula to the normal state dHvA data; whilst the fit is good within the normal state, the dHvA oscillations are attenuated with decreasing field much more rapidly than the LK formula predicts once the vortex state is entered. On entering the vortex state, the scattering rate appears to increase from the constant value observed in the normal state.

Studies in other type II superconductors [15-17] have shown that dHvA oscillations persist below $B_{\mathrm{c} 2}$ and that there is a suppression of their amplitude in the vortex state which can be interpreted as an additional energy broadening of the Landau levels. Theoretical models [19] suggest that the effect of the vortex state can be

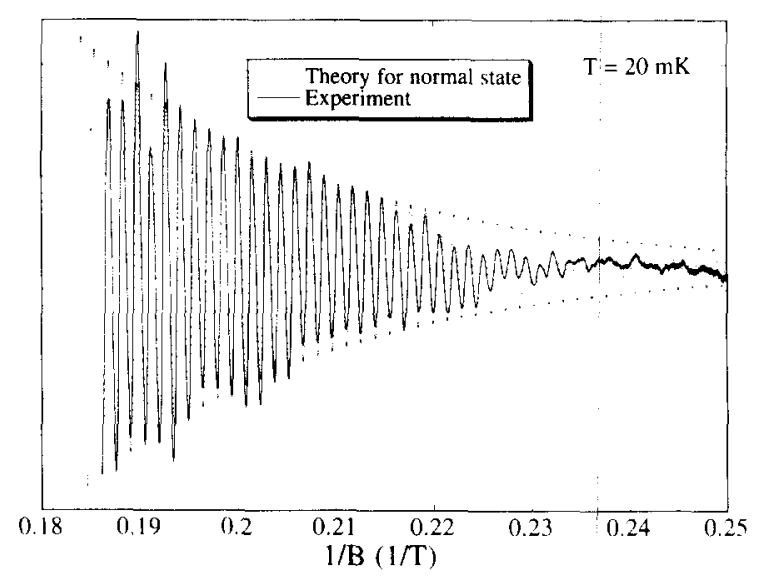

Fig. 5. The de Haas-van Alphen signal from $\kappa-(\mathrm{ET})_{2} \mathrm{Cu}(\mathrm{NCS})_{2}$ at $20 \mathrm{mK}$ and $\theta=29^{\circ}$ with the background subtracted [18] (solid line). The dotted line is the signal predicted by the LK formula fitted to normal state data; below $B_{\mathrm{c} 2}(\sim 4.6 \mathrm{~T})$, the data are attenuated more quickly than predicted by the LK formula. 
incorporated into an additional field-dependent scattering rate which is proportional to the square of the superconducting gap, $\Delta$. Although the model has been used to successfully account for observations in $\mathrm{NbSe}_{2}[15,16]$ and $\mathrm{A} 15$ superconductors [16,17], at present detailed comparisons with the data for $\mathrm{K}$ - $(\mathrm{ET})_{2} \mathrm{Cu}(\mathrm{NCS})_{2}$ are difficult due to problems in measuring $B_{\mathrm{c} 2}$ caused by the broad superconducting-normal transition. The short coherence length of $\mathrm{K}$-(ET) ${ }_{2} \mathrm{Cu}(\mathrm{NCS})_{2}$ means that the superconducting properties are probably sensitive to spatial inhomogenities in the system $[2,20]$, and this may be responsible for the broadening of features such as the superconducting anomaly in the heat capacity [21]. Further studies are under way using more sensitive apparatus; it is hoped that besides revealing the angular dependence of $\Delta$, the measurements will provide information about the cause of the broadened transition and a novel method of determining the bulk average value of $B_{\mathrm{c} 2}[18]$.

\section{Angle-dependent magnetoresistance oscillations}

The technique of observing MR as a sample is rotated in constant magnetic field has become a powerful tool for deducing the FS topology of organic metals. Both (1D) and 2D sections of FS can cause angle-dependent magnetoresistance oscillations (AMRO) via mechanisms summarised below; in this discussion, $\theta$ is defined as the angle between the field and the normal to the $2 \mathrm{D}$ planes.

1D AMRO result directly from a resonance between the field direction and the wave vector of each of the Fourier components of the corrugation of the quasi-1D FS sheets [22-24]; the resonances take the form of the dips in the MR periodic in $\tan (\theta)[22,23]$. The conductivity depends dramatically on whether electrons run across or along each corrugation of the quasi-1D sheet because the velocity-velocity correlation function is fundamentally different in each case [24]. When the magnetic field moves out of the quasi-1D sheets, the resonances spread out in angle due to purely geometrical considerations; the periodicity is proportional to $1 / \cos \left(\phi-\phi_{0}\right)$, $\phi$ being the azimuthal angle describing the plane of rotation of the field $[22,23]$. The AMRO also weaken, because the electron-path becomes curved, resulting in greater averaging of the velocities [24]. The strength of each dip is related to the amplitude of a particular Fourier component of the corrugation of the quasi-1D sheets [24].

We have thus far described the band-structures of the ET salts as quasi-2D. However, weak inter-plane coupling is important, and causes a slight warping of the FS in the direction perpendicular to the 2D plane; i.e. the hole pocket in Fig. 2 is a warped cylinder. AMRO peaks due to warped quasi-2D FS have been treated in several theoretical works $[25,26]$ and are connected with the vanishing of the electronic group velocity perpendicular to the 2D layers. The angles $\theta_{i}$ at which the maxima occur are given by $b^{\prime} k_{\|} \tan \left(\theta_{i}\right)=\pi\left(\mathrm{i} \pm\left(-\frac{1}{4}\right)\right)+A(\phi)$, where the signs + and - correspond to positive and negative $\theta_{i}$ respectively, $b^{\prime}$ is the effective inter-plane spacing, $k_{\|}$is the maximum Fermi wave vector projection on the plane of rotation of the field and $i= \pm 1$, $\pm 2 \ldots$ [25]. Here positive $i$ corresponds to $\theta_{i}>0$ and negative $i$ to $\theta_{i}<0$. The gradient of a plot of $\tan \left(\theta_{i}\right)$ against $i$ may thus be used to find one of the dimensions of the FS, and, if the process is repeated for several planes of rotation, the complete FS may be mapped [25, 27]. $A(\phi)$ is a function of the plane of rotation of the field, determined by the inclination of the plane of warping; hence this may also be found [25].

Both types of AMRO are illustrated using data from $\alpha$-(ET) ${ }_{2} \mathrm{KHg}(\mathrm{SCN})_{4}$ [28]. Fig. 6 shows MR data at $15 \mathrm{~T}$; a series of AMRO are observed as $\theta$ is varied. The minima are very sharp and are periodic in $\tan (\theta)$ at each

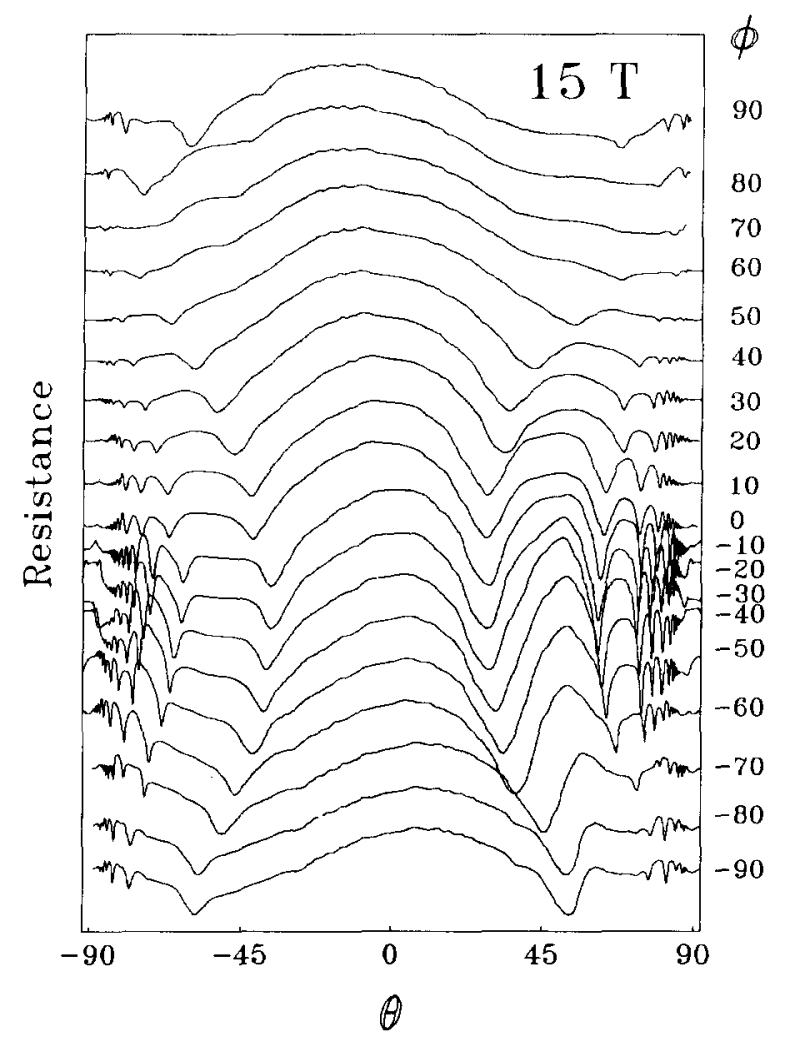

Fig. 6. AMRO of an $\alpha-(\mathrm{ET})_{2} \mathrm{KHg}(\mathrm{SCN})_{4}$ crystal at $1.5 \mathrm{~K}$ and $15 \mathrm{~T}$ as a function of tilt angle $\theta$ and azimuthal angle $\phi$. The traces are offset for clarity $[24,28]$. 
azimuthal angle. However, this periodicity varies as a function of $\phi$, being proportional to $1 / \cos \left(\phi-\phi_{0}\right)$, with $\phi_{0}=21 \pm 2^{\circ}(\phi=0$ represents rotation about the $a$-axis and positive $\phi$ represent angular displacement of the rotation axis in the 2D ac plane towards the $c$-axis). Note also that the amplitude of the AMRO varies with $\phi$. All of these features are characteristic of the presence of a ID section of FS, as discussed above; in this case, the presence of a warped quasi-1D FS tilted by $21^{\circ}$ with respect to the $b * c$ plane is thought to be due to reordering of the quasi-2D FS by a spin-density wave (SDW) $[22,28]$.

Fig. 7 shows AMRO at fixed $\phi$ : as the applied field increases, the dips in the MR disappear and new peaks appear. The peaks at high fields have a much more isotropic dependence on $\phi$, and are characteristic of AMRO due to the presence of a quasi-2D FS cylinder, as discussed above [28]. This result illustrates that the magnetic field has induced a change in the character of the FS form 1D to 2D; this is thought to be associated with the destruction of the SDW by the external field $[28,29]$. Similar changes in the AMRO, from 1D to 2D character, are observed when $\alpha-(\mathrm{ET})_{2} \mathrm{KHg}(\mathrm{SCN})_{4}$ is warmed up through the SDW ordering temperature [29].

\section{Effective masses and cyclotron resonance}

The effective masses of carriers in ET charge-transfer salts measured using the $\mathrm{SdH}$ and $\mathrm{dHvA}$ effects are known to be much larger than those predicted by bandstructure calculations, suggesting that many body effects are important $[5,30]$. Within the theory of interacting Fermions a number of quasi-particle "masses" can be defined, reflecting the differing dynamical behaviour in-

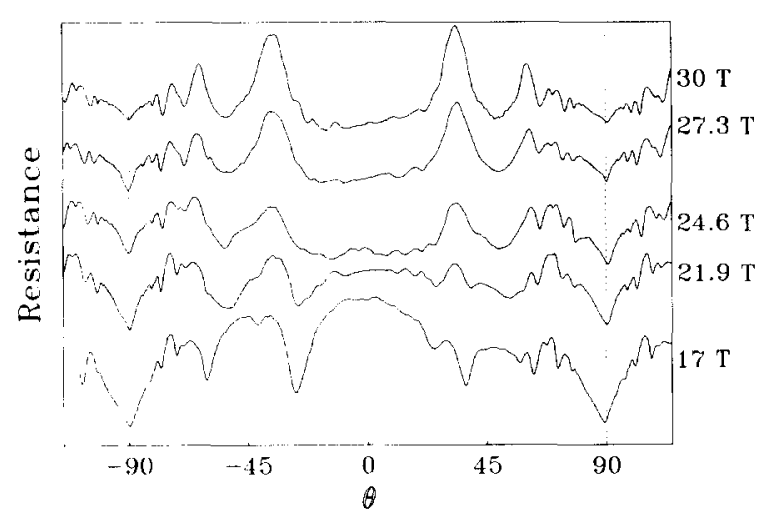

Fig. 7. $A M R O$ in $\alpha-(E T)_{2} \mathrm{KHg}(\mathrm{SCN})_{4}$ at $1.5 \mathrm{~K}$. The sharp dips seen in $17 \mathrm{~T}$ are seen at all lower fields, but disappear in the range $22-25 T$ to be replaced by peaks $[24,28]$. volved in various physical properties [31]. The simplest is the bare band mass $m_{\mathrm{b}}$, which is derived from single particle band-structure calculations. This is close in size to the optical mass $m_{\text {opt }}$ which can be probed by an optical measurement of the intraband plasma response [31]. The effective mass $m^{*}$ occurs in the thermodynamic density of states, and may crudely be thought of as arising from a quasi-particle displacing other quasiparticles as it moves through a medium. The backflow of quasi-particles leads to a contribution to the effective mass $m^{*}$. The dynamical mass $m_{\lambda}$ is the mass which would be observed in the absence of quasi-particle interactions; it represents $m_{\mathrm{b}}$ renormalised only by electron-phonon interactions, whereas $m^{*}$ contains contributions from both quasi-particle interactions and electron-phonon interactions. The temperature dependence of phenomena such as the $\mathrm{SdH}$ and dHvA effects determines the effective mass $m^{*}$ whereas a microwave cyclotron resonance (CR) experiment measures $m_{\lambda}[30,31]$.

A typical $\mathrm{CR}$ spectrum is shown in Fig. 8 for $\alpha$ $(\mathrm{ET})_{2} \mathrm{NH}_{4} \mathrm{Hg}(\mathrm{SCN})_{4}$ [32]. Besides the fundamental $\mathrm{CR}$ at $2.5 \mathrm{~T}$, there are a number of other features, interpreted as higher harmonics; these are explained in detail elsewhere [32]. The important point to note is that the mass measured from $\mathrm{CR}\left(m_{\lambda}\right)$ is a factor $\sim 2$ smaller than that derived from the $\mathrm{SdH}$ effect $\left(m^{*}\right)[10,33]$; this is a measure of the degree of renormalisation introduced by quasi-particle interactions.

Various quasi-particle masses have been derived for the $\alpha$-orbit of $\kappa$-(ET) ${ }_{2} \mathrm{Cu}(\mathrm{NCS})_{2}$ at ambient pressure [11]; $m_{\mathrm{b} \alpha}=0.64 m_{\mathrm{e}}$ was obtained from a fit of band-structure calculations to low temperature optical data [11], CR experiments give a value of $m_{\lambda \alpha}=1.18 m_{\mathrm{c}}$ [33] and $m_{\alpha}^{*}=3.5 m_{\mathrm{e}}$ was measured using the SdH effect (Fig. 4)

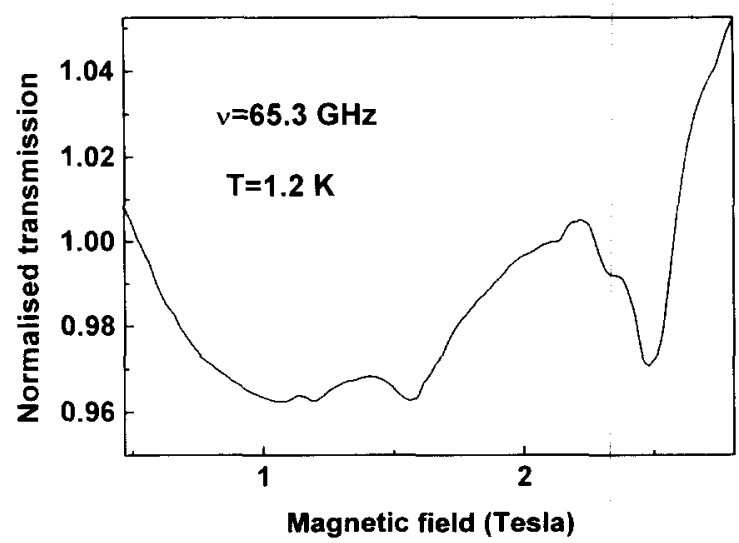

Fig. 8. Swept-field cyclotron resonance data from $\alpha$ $(\mathrm{ET})_{2} \mathrm{NH}_{4} \mathrm{Hg}(\mathrm{SCN})_{4}$ (temperature and frequency shown in figure) [32]. 
[11]. The latter value represents an enhancement of a factor of $\sim 3$ over $m_{\lambda \alpha}$. The quasi-particle interactions thus appear to give a larger renormalisation of the quasiparticle mass than do the electron-phonon interactions $\left(m_{\lambda \alpha} / m_{\mathrm{b} \alpha}\right.$ is a factor $\sim 2$ smaller than $m_{\alpha}^{*} / m_{\lambda \alpha}$ ). No CR data corresponding to the $\beta$-orbit have yet been observed. However, the ratios of the effective masses to the band masses are similar for the two orbits [11], indicating that the renormalisation of the bare band mass is relatively uniform over the whole FS.

The effects of many body interactions therefore appear to be very important in giving the large effective masses in ET charge-transfer salts $[5,11]$. It also seems that large effective masses are a prerequisite for superconductivity in ET salts (Fig. 4) and that the mechanisms primarily responsible for producing the mass enhancement are due to interactions between quasi-particles, with electron-phonon interactions playing a smaller role [11]. The initial strong decrease in effective mass with pressure seen in Fig. 4 could therefore represent suppression of a component of the quasi-particle interactions which depends critically on the bandwidth; the bandwidth of course increases with pressure.

\section{Concluding remarks}

We have reviewed some of the physics which can be done with ET salts in high magnetic fields. Many similarities between the ET salts and high $T_{\mathrm{c}}$ superconductors may have become apparent $[2,3]$; e.g. $2 \mathrm{D}$ band-structure with weak inter-plane coupling; low carrier densities, implying less screening, leading to the importance of quasi-particle interactions; the possibilities of both antiferromagnetism and superconductivity; heavy effective masses $\left(\sim 2-5 m_{\mathrm{e}}\right)$ leading to small Fermi velocities $\left(\sim 10^{5} \mathrm{~ms}^{-1}\right)$ and Fermi energies $(\sim 0.1 \mathrm{eV})$. Many of these properties are also shared with heavy Fermion systems [5]. The attraction of ET salts is that they contain all of this interesting physics but may be studied using reasonable fields $(10-50 \mathrm{~T})$ and temperatures $(0.08-5 \mathrm{~K})$ [6]. They may well, therefore, be the first systems on which theoretical approaches to high $T_{\mathrm{c}}$ superconductors or heavy Fermion systems are tested.

\section{Acknowledgements}

This work is supported by EPSRC, the EU and the Royal Society (UK). The Nijmegen High Field Magnet Laboratory is supported by FOM and the EC Large Installations Plan. We thank our many collaborators in Boston, Bristol, Cambridge, Chernogolovka, Leuven,
London, Moscow, Nijmegen, Oxford and Sheffield for invaluable help, discussions and access to unpublished data.

\section{References}

[1] For a review, see in: Proc. Int. Conf. on Science and Technology of Synthetic Metals, Göteborg, Sweden, August 12-18 (1992); Synth. Met. 56-8 (1993).

[2] T. Ishiguro and K. Yamaji, Organic Superconductors (Springer, Berlin, 1990).

[3] V.Z. Kresin and W.A. Little (eds.), Organic Superconductivity (Plenum Press, New York, 1990).

[4] H. Urayama, H. Yamochi, G. Saito, K. Nozawa, T. Sugano, M. Kinoshita, S. Sato, K. Oshima, A. Kawamoto and J. Tanaka, Chem. Lett. (1988) 55.

[5] N. Toyota, E.W. Fenton, T. Sasaki and M. Tachiki, Solid State Commun. 72 (1989) 859.

[6] J. Singleton, F.L. Pratt, M. Doporto, J.M. Caulfield, S.O. Hill, T.J.B.M. Janssen, I. Deckers, G. Pitsi, F. Herlach, W. Hayes, J.A.A.J. Perenboom, M. Kurmoo and P. Day, Physica B 184 (1993) 470.

[7] T. Sasaki, H. Sato and N. Toyota, Physica C 185-189 (1991) 2687; Solid State Commun. 76 (1990) 507.

[8] C.-P. Heidmann, H. Müller, W. Biberacher, K. Neumaier, C. Probst, K. Andres, A.G.M. Jansen and W. Joss, Synth. Met. 41-43 (1991) 2029.

[9] J. Caulfield, J. Singleton, F.L Pratt, M. Doporto, W. Lubczynski, W. Hayes, M. Kurmoo, P. Day, P.T.J. Hendriks and J.A.A.J. Perenboom, Synth. Met. 61 (1993) 63.

[10] M. Doporto, F.L. Pratt, J. Singleton, M. Kurmoo and W. Hayes, Phys. Rev. Lett. 69 (1992) 991.

[11] J. Caulfield, W. Lubczynski, F.L. Pratt, J. Singleton, D.Y.K. Ko, W. Hayes, M. Kurmoo and P. Day, J. Phys.: Condens. Matter 6 (1994) 2911.

[12] W. Lubczynski, J. Caulfield, J. Singleton, F.L. Pratt, W. Hayes, M. Kurmoo and P. Day. In: Proc. Int. Conf. on Synthetic Metals (ICSM 94), Seoul (1994); Synth. Met., to be published.

[13] J. Wosnitza, G.W. Crabtree, H.H. Wang, U. Geiser, J.M. Williams and K.D. Carlson, Phys. Rev. B. 45 (1992) 3018.

[14] A.G. Swanson, J.S. Brooks, H. Anzai, N. Konoshita, M. Tokumoto and K. Murata, Solid State Commun. 73 (1990) 353.

[15] J.E. Graebner and M. Robbins, Phys. Rev. Lett. 36 (1976) 433.

[16] R. Corcoran, P. Meeson, Y. Onuki, P-A. Probst, M. Springford and T. Takita, Physica B 194-196 (1994) 1573.

[17] N. Harrison, S.M. Hayden, P. Meeson, M. Springford, P.J. van der Wel and A.A. Menovsky, Phys. Rev. B., to be published.

[18] P.J. van der Wel, J. Caulfield, J. Singleton, R. Corcoran, S.M. Hayden, M. Springford, W. Hayes, M. Kurmoo and P. Day, in: Proc. Int. Conf. on Materials and Mechanics of Superconductivity and High Temperature Superconductivity IV, Grenoble (1994); Physica C, to be published. 
[19] K. Maki, Phys. Rev. B 44 (1991) 2861; A. Wasserman and M. Springford, Physica B 194-196 (1994) 1801.

[20] See, for example F. Hellman and T.H. Geballe, Phys. Rev. B 36 (1987) 107.

[21] J.E. Graebner, R.C. Haddon, S.V. Chichester and S.H. Clarum, Phys. Rev. B 41 (1990) 4808.

[22] M.V. Kartsovnik, A.E. Kovalev and N.D. Kushch, J. Phys. I France 3 (1993) 1187; M.V. Kartsovnik, A.E. Kovalev, V.N. Laukhin and S.I. Pesotskii, J. Phys. I France 2(1992) 223.

[23] A.G. Lebed' and P. Bak, Phys. Rev. Lett. 63 (1989) 1315; G. Montambaux and P.B. Littlewood, Phys. Rev. Lett. 62 (1989) 953; V.M. Yakavenko, Phys. Rev. Lett. 68 (1992) 3607; M.J. Naughton, O.H. Chung, M. Chaparaba, X. Bu and P. Coppens, Phys. Rev. Lett. 67 (1991) 3712; T. Osada, S. Kagoshima and N. Miura, Phys. Rev. B 46 (1992) 1812.

[24] S.J. Blundell, to be published.

[25] M.V. Kartsovnik, V.N. Laukhin, S.I. Pesotskii, I.F. Schegolev and V.M. Yakovenko, J Phys. I France 2 (1992) 89.

[26] K. Yamaji, J. Phys. Soc. Japan 58 (1989) 1520.

[27] M. Doporto, J. Singleton, F.L. Pratt, J. Caulfield, W.
Hayes, J.A.A.J. Perenboom, I. Deckers, G. Pitsi, M. Kurmoo and P. Day, Phys. Rev. B 49 (1994) 3934.

[28] J. Caulfield, J. Singleton, P.T.J. Hendriks, J.A.A.J. Perenboom, F.L. Pratt, M. Doporto, W. Hayes, M. Kurmoo and P. Day, J. Phys.: Condens. Matter 6 (1994) L155.

[29] A.E. Kovalev, M.V. Karisovnik, R.P. Shibaeva, L.P. Rozenberg, I.F. Schegolev and N.D. Kusch, Solid State Commun. 89 (1994); 575; T. Sasaki and N. Toyota, Phys. Rev. B 49 (1994) 10120.

[30] J. Singleton, F.L. Pratt, M. Doporto, W. Hayes, T.J.B.M. Janssen, J.A.A.J. Perenboom, M. Kurmoo and P. Day, Phys. Rev. Lett. 68 (1992) 2500.

[31] K.F. Quader, K.S. Bedell and G.E. Brown, Phys. Rev. B 36 (1987) 156; G.D. Mahan, Many Particles Physics (Plenum Press, New York, 1990); A.J. Leggett, Ann. Phys. 46 (1968) 76.

[32] S. Hill, J. Singleton, J. van Bentum, A. Wittlin, W. Hayes, M. Kurmoo and P. Day, in: Proc. Int. Conf. on Synthetic Metals, Seoul, Korea, July (1994); Synth. Met., to be published.

[33] S. Hill, J. Singleton, F.L. Pratt, M. Doporto, W. Hayes, T.J.B.M. Janssen, J.A.A.J. Perenboom, M. Kurmoo and P. Day, Synth. Met. 56 (1993) 2566. 